\title{
INTERIOR NUMERICAL APPROXIMATION OF BOUNDARY VALUE PROBLEMS WITH A DISTRIBUTIONAL DATA
}

\author{
IVO BABUŠKA AND VICTOR NISTOR
}

\begin{abstract}
We study the approximation properties of a harmonic function $u \in H^{1-k}(\Omega), k>0$, on relatively compact sub-domain $A$ of $\Omega$, using the Generalized Finite Element Method. For smooth, bounded domains $\Omega$, we obtain that the GFEM-approximation $u_{S}$ satisfies $\left\|u-u_{S}\right\|_{H^{1}(A)} \leq C h^{\gamma}\|u\|_{H^{1-k}(\Omega)}$, where $h$ is the typical size of the "elements" defining the GFEM-space $S$ and $\gamma \geq 0$ is such that the local approximation spaces contain all polynomials of degree $k+\gamma+1$. The main technical result is an extension of the classical super-approximation results of Nitsche and Schatz [?] and, especially, [?]. It turns out that, in addition to the usual "energy" Sobolev spaces $H^{1}$, one must use also the negative order Sobolev spaces $H^{-l}, l \geq 0$, which are defined by duality and contain the distributional boundary data.
\end{abstract}

\section{Contents}

Introduction 1

1. Preliminaries 3

2. The Generalized Finite Element Method 5

3. Interior estimates for the GFEM 7

4. Approximate solution of the Laplace equation with distribution boundarv conditions using the GFEM

5. Polvnomial local approximation spaces

20

\section{INTRODUCTION}

To motivate our results, let us consider the boundary value problem

$$
\begin{cases}\Delta u=0 & \text { on } \Omega \\ \frac{\partial u}{\partial \nu}=g & \text { on } \Gamma:=\partial \Omega\end{cases}
$$

where $\Omega$ is a smooth, bounded domain in $\mathbb{R}^{n}, \partial \Omega$ is the boundary of $\Omega$ and $g \in$ $H^{r-3 / 2}(\partial \Omega), r \in \mathbb{R}$. The case $r>3 / 2$ was extensively studied. Here we are interested mainly in the case $r \leq 3 / 2$. We are looking for a solution $u \in H^{r}(\Omega)$.

For $r>3 / 2$, the boundary values (or traces) $\left.u\right|_{\partial \Omega}$ and $\left.\partial_{\nu} u\right|_{\partial \Omega}$ are defined classically, because the restriction to the boundary extends by continuity to a map $H^{r}(\Omega) \ni u \rightarrow \partial_{\nu} u \in H^{r-3 / 2}(\partial \Omega)$, see [?, ?] for example. For $r \leq 3 / 2$, this is no longer true, but then one takes advantage of the fact that $u$ satisfies an elliptic equation, so it is still possible to define $\partial_{\nu} u \in H^{r-3 / 2}(\partial \Omega)$, see [?, ?]. We can

I. Babuška was partially supported by NSF Grant DMS 0341982. V. Nistor was partially supported by NSF Grants DMS 991981 and 0200808. Manuscripts available from http://www.math.psu.edu/nistor/. 
assume, without loss of generality, that $\Omega$ is connected. It is not difficult to prove that a solution $u$ of Equation (11) exists for any $g$ such that $\langle g, 1\rangle=0$ and that this solution satisfies

$$
\|u\|_{H^{r}(\Omega)} \leq C\|g\|_{H^{r-3 / 2}(\partial \Omega)},
$$

with a constant $C$ that may depend on $r$ but is independent of $g$. This result will be discussed in detail in [?], where more references will be given. (This result will be used in this paper only as a motivation for our work.)

Recall that $A \Subset \Omega$ means that $A \subset \Omega$ is bounded and $\partial A$ and $\partial \Omega$ are disjoint. If $A \Subset \Omega$ is an open subset, then $u$ satisfying (10 will be smooth on $A$, regardless of what $r$ is, and

$$
\|u\|_{H^{m}(A)} \leq C\|u\|_{H^{r}(\partial \Omega)},
$$

with a constant $C$ that depends on $A, \Omega, r$, and $m$, but is independent of $u$ satisfying $\Delta u=0$. An important problem, with potential practical applications, is to approximate on $A$ the solution $u$ of Equation (11).

In this paper, we prove several results on the approximation of the solution $u$ on subsets $A \Subset \Omega$. The main result of the paper, Theorem 4.8 then gives in particular that the solution $u$ of the boundary value problem (10) satisfies

$$
\left\|u-u_{S}\right\|_{H^{1}(A)} \leq C h^{\gamma}\|u\|_{H^{1-k}(\Omega)},
$$

where $u_{S} \in S$ is the Galerkin approximation and $S$ is the Generalized Finite Element Space associated to a partition of unity $\left\{\phi_{j}\right\}$ subordinated to a covering $\left\{\omega_{j}\right\}$ of $\Omega$ satisfying Assumptions A-D of Section 2 provided that our local approximation spaces contain all of polynomials of degree $1+\gamma+k, \gamma \geq 0$. We stress that our results require not just the energy Sobolev space $H^{1}$, but also negative order Sobolev spaces $H^{-l}$, defined in this paper as the duals of $H^{l}, l \in \mathbb{Z}_{+}$. One of the main reasons for the need to considere the negative order Sobolev spaces is that the solution $u$ is not in $H^{1}(\Omega)$ but in $H^{1-k}(\Omega)$, in general. Moreover, even if we approximate the boundary data $g$ and the solution $u$ with functions in $H^{1}$, then the norm on a negative order Sobolev space will still have to appear in the estimate of the error.

Here is now a brief description of the contents of the paper. We continue to assume that $\Omega$ is bounded and connected, but we no longer assume that $\Omega$ is smooth, except when explicitly mentioned. In Section 1 we set up the notation and we establish our conventions on Sobolev spaces. Section 2 contains a quick review of the necessary definitions involving the Generalized Finite Element Method (GFEM) and their variants used in this paper [?, ?, ?]. This section also contains the assumptions that we make on our covering and partition of unity used to define the GFEM-space $S$. The space $S$ will contain our approximate solution to the boundary value problem. The following section, Section 3 contains the calculations necessary to establish our interior estimates. Our approach follows, to a certain extend, that in the article of Nitsche and Schatz [?], relying also from Wahlbin's survey article [?]. The main differences between our paper and [?, ?] are due mostly to the fact that several assumptions from these papers are not fully satisfied in our approach. As in these articles, the main step is a super-approximation property, Proposition 3.6. The proof in [?, ?] cannot be used to obtain Proposition 3.6 because the property " $\partial^{\alpha} u=0$ if $|\alpha|$ is large," is not satisfied in general for $u \in S$. For the results of Section 4 , we assume that $\Omega$ is smooth. Then we extend the definitions of 
the Galerkin approximation $u_{S} \in S$ and of the form $B(w, v):=\int_{\Omega} \nabla u(x) \cdot \nabla v(x) d x$ to the case when $v \in H^{k-1}(\Omega)$ is arbitrary and $u \in H^{1-k}(\Omega)$ can be written as $u=u_{1}+u_{2}$, where $\Delta u_{1}=0$ in distributions sense and $u_{2} \in H^{1}(\Omega)$. In what follows, $1-k$ will play the role of $r$ above. Several estimates for $u$ and its approximation $u_{S}$ are established in this section, including the main theorem, Theorem 4.8 (whose main conclusion was repeated in Equation (44) above). The last section, Section 5 contains a proof that, for a domain with piecewise $C^{1}$-boundary, we can construct a family of partitions of unity with typical size of supports $h \rightarrow 0$ that satisfies Assumptions A-D for a fixed choice of the other values of the parameters (i.e., of $A$, $B, C_{j}, \kappa, m$, and $\left.\sigma\right)$. For this construction we assume that the local approximation spaces are $\Psi_{j}=\mathcal{Q}_{k}$, the space of polynomials of degree at most $k$. In particular, the various assumptions made in the results proved in the preceding sections, are satisfied for this family of partition of unity, and hence our results are not empty for domains with piecewise smooth boundary. By contrast, it is not possible to find a family of partitions of unity as above for domains with cusps, see Remark 5.7. For suitable $g$, we plan to perform some concrete numerical simulations in a future paper.

We shall write $x:=y$ if $x$ is defined by $y$.

The second named author thanks G. Grubb, A. Schatz, and L. Wahlbin for some useful references. A. Schatz has also made some useful comments on an earlier version of the manuscript, for which we are greatful.

\section{Preliminaries}

We begin by fixing the notation and terminology.

1.1. Preliminary notation. We denote by $\mathbb{R}$ the set of real numbers and by $\mathbb{C}:=\{a+b \imath, a, b \in \mathbb{R}\}$ the set of complex numbers. Also, $\mathbb{N}=\{1,2, \ldots\}$ and $\mathbb{Z}_{+}=\{0\} \cup \mathbb{N}$. Let $x \cdot y:=x_{1} y_{1}+x_{2} y_{2}+\ldots+x_{n} y_{n}$ be the inner product of two vectors $x, y \in \mathbb{R}^{n}$. We shall denote by

$$
\hat{f}(y):=\int_{\mathbb{R}^{n}} e^{-i y \cdot x} f(x) d x
$$

the Fourier transform of $f$, as usual. By $L^{2}(\Omega)$ we shall denote the space of square integrable functions $f: \Omega \rightarrow \mathbb{C}$, for any domain $\Omega \subset \mathbb{R}^{n}$.

Let $s \geq 0$. Then $H^{s}\left(\mathbb{R}^{n}\right)$ is the space of functions $f \in L^{2}\left(\mathbb{R}^{n}\right)$ such that

$$
\|f\|_{H^{s}\left(\mathbb{R}^{n}\right)}^{2}:=(2 \pi)^{-n} \int_{\mathbb{R}^{n}}\left(1+|y|^{2}\right)^{s}|\hat{f}(y)|^{2} d y<\infty .
$$

Here $|y|=\sqrt{y_{1}^{2}+\ldots+y_{n}^{2}}$, if $y=\left(y_{1}, \ldots, y_{n}\right) \in \mathbb{R}^{n}$. Let $\Omega \subset \mathbb{R}^{n}$, then $H^{s}(\Omega)$, $s \geq 0$, denotes the restrictions to $\Omega$ of functions $f \in H^{s}\left(\mathbb{R}^{n}\right)$, that is,

$$
H^{s}(\Omega):=\left\{\left.f\right|_{\Omega}, f \in H^{s}\left(\mathbb{R}^{n}\right)\right\} .
$$

The norm on $H^{s}(\Omega)$ is then $\|h\|_{H^{s}(\Omega)}:=\inf \|f\|_{H^{s}\left(\mathbb{R}^{n}\right)}$, the infimum being taken over all functions $u \in H^{s}\left(\mathbb{R}^{n}\right)$ such that $\left.f\right|_{\Omega}=h$. When $s$ is a positive integer and $\Omega$ is a nice domain (Lipschitz, for example), the norm $\|v\|_{H^{s}(\Omega)}$ is equivalent to the usual norm

$$
\|h\|_{s}^{2}=\sum_{|\alpha| \leq s}\left\|\partial^{\alpha} h\right\|_{L^{2}(\Omega)}^{2}
$$


where $\alpha=\left(\alpha_{1}, \ldots, \alpha_{n}\right) \in \mathbb{Z}_{+}^{n},|\alpha|:=\alpha_{1}+\alpha_{2}+\ldots+\alpha_{n}$, and $\partial^{\alpha}:=\partial_{1}^{\alpha_{1}} \partial_{2}^{\alpha_{2}} \ldots \partial_{n}^{\alpha_{n}}$, as usual. The space $H_{0}^{s}(\Omega)$ is defined as the closure of $\mathcal{C}_{\mathrm{c}}^{\infty}(\Omega)$ in $H_{0}^{s}(\Omega)$.

1.2. Distributions. Let $B_{R}(0)$ denote the open ball of radius $R$ centered at the origin. Also, let $\mathcal{C}_{\mathrm{c}}^{\infty}\left(\mathbb{R}^{n}\right)$ be the set of infinitely differentiable, complex valued functions that vanish outside the ball $B_{R}(0)$, for some large $R>0$. A linear map $u: \mathcal{C}_{\mathrm{c}}^{\infty}\left(\mathbb{R}^{n}\right) \rightarrow \mathbb{C}$ is called a distribution on $\mathbb{R}^{n}[?, ?, ?]$ if, for any $R>0$, there exists $m \in \mathbb{Z}_{+}=\{0,1,2, \ldots\}$ and $C>0$ such that

$$
|u(\phi)| \leq C \sum_{|\alpha| \leq m}\left\|\partial^{\alpha} \phi\right\|_{L^{\infty}}, \quad \text { if } \phi \in \mathcal{C}_{\mathrm{c}}^{\infty}\left(\mathbb{R}^{n}\right) \text { and } \phi=0 \text { outside } B_{R}(0) .
$$

This definition does not exclude the case when larger and larger values of $m$ have to be chosen as $R \rightarrow \infty$, and in fact this situation actually occurs in specific examples. The set of distributions on $\mathbb{R}^{n}$ will be denoted $\mathcal{D}^{\prime}\left(\mathbb{R}^{n}\right)$.

We now fix more notation and terminology. If $f$ is a function, then the closure of the set $\{f \neq 0\}$ is called the support of $f$ and will be denoted $\operatorname{supp}(f)$. We shall also write $\langle u, \phi\rangle:=u(\phi)$ for the value of the distribution $u$ on the function $\phi \in \mathcal{C}_{\mathrm{c}}^{\infty}\left(\mathbb{R}^{n}\right)$. Therefore, any $\phi \in \mathcal{C}_{\mathrm{c}}^{\infty}(\Omega)$ has compact support. The support of a distribution $u$ is the smallest closed set $F$ such that $\langle u, \phi\rangle=0$ for any $\phi \in \mathcal{C}_{\mathrm{c}}^{\infty}\left(\mathbb{R}^{n} \backslash F\right)$.

Here are some examples of distributions.

Example 1.1. If $f$ is a measurable function on $\mathbb{R}^{n}$ that is integrable on any closed ball in $\mathbb{R}^{n}$ (i.e., it is locally integrable, or $f \in L_{\text {loc }}^{1}\left(\mathbb{R}^{n}\right)$ ), then we can define

$$
\langle f, \phi\rangle:=\int_{\mathbb{R}^{n}} f(x) \phi(x) d x,
$$

for any $\phi \in \mathcal{C}_{\mathrm{c}}^{\infty}\left(\mathbb{R}^{n}\right)$. Thus any $f \in L_{\text {loc }}^{1}\left(\mathbb{R}^{n}\right)$ defines a distribution on $\mathbb{R}^{n}$, that is, $L_{\text {loc }}^{1}\left(\mathbb{R}^{n}\right) \subset \mathcal{D}^{\prime}\left(\mathbb{R}^{n}\right)$. In this situation we shall say that our distribution $f$ is, in fact, a locally integrable function, or, that our distribution is defined by a locally integrable function.

The following two examples are relevant for the discussion of concentrated loads and moments.

Example 1.2. The Dirac measure at $a \in \mathbb{R}^{n}$ is the distribution $\delta_{a}$ defined by

$$
\left\langle\delta_{a}, \phi\right\rangle:=\phi(a) .
$$

An explicit calculation shows that $\delta_{a} \in H^{-n / 2-\epsilon}\left(\mathbb{R}^{n}\right)$ and $\left\|\delta_{a}\right\|_{H^{-n / 2-\epsilon}\left(\mathbb{R}^{n}\right)} \rightarrow \infty$ as $\epsilon \rightarrow 0$ as $\epsilon^{1 / 2}$.

Example 1.3. The derivatives $\partial^{\alpha} u$ of a distribution $u$ are defined by

$$
\left\langle\partial^{\alpha} u, \phi\right\rangle:=(-1)^{|\alpha|}\left\langle u, \partial^{\alpha} \phi\right\rangle .
$$

We now define the negative index Sobolev spaces. Thus, the space $H^{-s}\left(\mathbb{R}^{n}\right), s \geq$ 0 , consists of all the distributions $u \in \mathcal{D}^{\prime}\left(\mathbb{R}^{n}\right)$ such that there exists $h \in L_{\text {loc }}^{1}\left(\mathbb{R}^{n}\right)$ satisfying

and

$$
\langle u, \hat{f}\rangle=\int_{\mathbb{R}^{n}} h(y) f(y) d y, \quad \text { for all } f \in \mathcal{C}_{\mathrm{c}}^{\infty}\left(\mathbb{R}^{n}\right)
$$

$$
\|u\|_{-s}^{2}:=(2 \pi)^{-n} \int_{\mathbb{R}^{n}}\left(1+|y|^{2}\right)^{s}|h(y)|^{2} d y<\infty .
$$


The reader will recognize that the condition above is analogous to the condition of Equation (6). The difference is that now we allow $s$ to take on negative values as well.

The following alternative definition of the negative order Sobolev spaces will be useful later on. One first checks directly using the Fourier inversion formula together with Plancherel's formula that

$$
\|u\|_{-s}^{2}:=\inf _{\phi} \frac{1}{\|\phi\|_{s}}\left|\int_{\mathbb{R}^{n}} u(x) \phi(x) d x\right|, \quad \text { where } u, \phi \in \mathcal{C}_{\mathrm{c}}^{\infty}\left(\mathbb{R}^{n}\right), v \neq 0 .
$$

Then $H^{-s}\left(\mathbb{R}^{n}\right)$ can be defined as the completion of $\mathcal{C}_{\mathrm{c}}^{\infty}\left(\mathbb{R}^{n}\right)$ in the norm given by the formula of Equation (12). In particular, we obtain that $H^{-s}\left(\mathbb{R}^{n}\right)$ is canonically isomorphic to the dual of $H^{s}\left(\mathbb{R}^{n}\right)$.

Similarly, for any open subset $\Omega \subset \mathbb{R}^{n}$, we define the space $H^{-s}(\Omega), s \geq 0$, as the dual of $H^{s}(\Omega)$.

$$
\|u\|_{H^{-s}(\Omega)}=\sup \frac{|(u, \phi)|}{\|\phi\|_{H^{s}(\Omega)}}, \quad 0 \neq \phi \in H^{s}(\Omega) .
$$

Our definition of negative order Sobolev spaces by duality follows [?, ?, ?], for example. Note however, that the negative order Sobolev spaces are often also defined by restriction from $\mathbb{R}^{n}$, as in [?, ?, ?], for example. The space of restrictions to $\Omega$ of distributions in $H^{-s}\left(\mathbb{R}^{n}\right)$ is the dual of $H_{0}^{s}(\Omega)$, and will be denoted $H_{0}^{-s}(\Omega)$. The spaces $H_{0}^{-s}(\Omega)=H_{0}^{s}(\Omega)^{*}, s \geq 0$, will also be used below.

When $\Omega=\mathbb{R}^{n}$, these two approaches yield the same spaces, but for general $\Omega$ they may lead to different "negative order" Sobolev spaces.

\section{The Generalized Finite Element Method}

We now recall a few basic facts about the Generalized Finite Element Method $[?, ?, ?]$. This method is especially convenient since it provides finite element spaces with high regularity. Most of the results of this section work for a general bounded open set $\Omega$, except the application to the boundary value problems, Subsection 2.2

2.1. Basic facts. We shall need the following slight generalization of a definition from $[?, ?]$ :

Definition 2.1. Let $\Omega \subset \mathbb{R}^{n}$ be an open set and $\left\{\omega_{j}\right\}$ be an open cover of $\Omega$ with no $\kappa+1$ of the sets $\omega_{j}$ having a non-empty intersection. Also, let $\left\{\phi_{j}\right\}$ be a partition of unity consisting of Lipschitz functions and subordinated to the covering $\left\{\omega_{j}\right\}$ (i.e., $\operatorname{supp} \phi_{j} \subset \omega_{j}$ ). If

$$
\left\|\partial^{\alpha} \phi_{j}\right\|_{L^{\infty}(\Omega)} \leq C_{k} /\left(\operatorname{diam} \omega_{j}\right)^{k}, \quad k=|\alpha| \leq m,
$$

then $\left\{\omega_{j}\right\}$ is called a $\left(\kappa, C_{0}, C_{1}, \ldots, C_{m}\right)$ partition of unity.

Assume also that linear subspaces $\Psi_{j} \in H^{m}\left(\omega_{j}\right)$ are given. These subspaces will be called local approximation spaces and are used to define the space

$$
S=S_{G F E M}:=\left\{\sum_{j} \phi_{j} v_{j}, v_{j} \in \Psi_{j}\right\},
$$

which will be called the GFEM-space.

A basic approximation property of the GFEM-spaces is the following Theorem from [?]. 
Theorem 2.2 (Babuška-Melenk). We use the notations and definitions of Definition 2.1 and after. Let $\left\{\phi_{j}\right\}$ be a $\left(\kappa, C_{0}, C_{1}\right)$ partition of unity. Also, let $v_{j} \in \Psi_{j}$, $u_{a p}:=\sum_{j} \phi_{j} v_{j} \in S$, and $d_{j}=\operatorname{diam} \omega_{j}$, the diameter of $\omega_{j}$. Then

$$
\begin{gathered}
\left\|u-u_{a p}\right\|_{L^{2}(\Omega)}^{2} \leq \kappa C_{0}^{2} \sum_{j}\left\|u-v_{j}\right\|_{L^{2}\left(\omega_{j}\right)}^{2} \quad \text { and } \\
\left\|\nabla\left(u-u_{a p}\right)\right\|_{L^{2}(\Omega)}^{2} \leq 2 \kappa \sum_{j}\left(\frac{C_{1}^{2}\left\|u-v_{j}\right\|_{L^{2}\left(\omega_{j}\right)}^{2}}{\left(d_{j}\right)^{2}}+C_{0}^{2}\left\|\nabla\left(u-v_{j}\right)\right\|_{L^{2}\left(\omega_{j}\right)}^{2}\right)
\end{gathered}
$$

For our method, we shall need to make some additional assumptions on the local approximation spaces $\Psi_{j}$, on the covering $\left\{\omega_{j}\right\}$, and on the partition of unity $\left\{\phi_{j}\right\}$. We shall denote by $d_{j}$ the diameter of $\omega_{j}$. Recall that we have assumed that $\Omega$ is connected, the general case being immediately reduced to this one. Unless otherwise mentioned, we shall make the following assumptions, for some fixed values of the parameters $A, B, C_{j}, \kappa, m, \sigma$, and $h$.

It follows that

$$
\sum_{\omega_{j}^{*} \subset \Omega} \phi_{j}=1 \quad \text { on } \Omega .
$$

In turn, Equation (16) implies the second condition in Assumption A. The covering $\left\{\omega_{j}\right\}$ will satisfy the following geometric assumption.

Assumption A. The sets $\omega_{j}$ are convex of diameters $d_{j} \leq h \leq 1$ and there exists a ball $\omega_{j}^{*}$ of diameter greater or equal $\sigma h$ whose closure is contained in $\omega_{j}$, for all $j$. Moreover, $\omega_{j}^{*} \subset \Omega$ if $\phi_{j}$ is not identically zero on $\Omega$.

This is a non-trivial assumption, see Section [5 Remark 5.7 The partition of unity will satisfy the following condition.

Assumption B. The family $\left\{\phi_{j}\right\}$ is a $\left(\kappa, C_{0}, C_{1}, \ldots, C_{m}\right)$ partition of unity such that

$$
\phi_{j}(x)=1 \quad \text { if } x \in \omega_{j}^{*} .
$$

It follows from our assumptions that the sets $\omega_{j}^{*}$ must be disjoint. Also, we have that

$$
\left\|\phi_{j}\right\|_{H^{l}(\Omega)} \leq C_{l} h^{-l}, \quad l=0,1, \ldots, m,
$$

by Definition 2.1 and Assumption A.

The local approximation spaces will satisfy:

Assumption C. The space $\Psi_{j}$ contains all (restrictions to $\omega_{j}$ of) first order polynomial functions and there exists $A>0$ such that

$$
\|w\|_{H^{l}\left(\omega_{j}\right)} \leq A\|w\|_{H^{l}\left(\omega_{j}^{*}\right)}
$$

for any $j$, any $w \in \Psi_{j}$, and any $0 \leq l \leq m$.

Finally, our last assumptions is the following "inverse assumption:"

Assumption D. There exists a constant $B>0$

$$
\|w\|_{H^{t}\left(\omega_{j}\right)} \leq B d_{j}^{t-s}\|w\|_{H^{s}\left(\omega_{j}\right)}
$$

for any $j$, any $w \in \Psi_{j}$, and any $0 \leq s \leq t \leq m$.

Assumptions $\mathrm{C}$ and $\mathrm{D}$ are satisfied, for example, if $\Psi_{j}$ is the space of polynomials of degree $\leq k-1$, for some fixed $k \geq 2$, see Section 5 
If $A \subset \Omega$ is an open subset, then $A$ satisfies Assumptions $\mathrm{B}, \mathrm{C}$, and $\mathrm{D}$, but not $\mathrm{A}$, in general. We shall hence need to single out the class of open subsets of $\Omega$ satisfying Assumption A.

Definition 2.3. An open subset $A \subset \Omega$ is called admissible if $\sum_{j \in J(A)} \phi_{j}=1$ on $A$, where $J(A)$ is the set of those indices $j$ such that $\omega_{j}^{*} \subset A$.

Remark 2.4. It is easy to see that $A \subset \Omega$ is an admissible open subset if, and only if, $A$ satisfies Assumption A. In particular, $\Omega$ is an admissible subset of itself (see also Equation (16)). Moreover, all our results on the set $\Omega$ extend without change (including the constants) to any admissible open subset $\Omega_{1} \subset \Omega$.

It is proved in Section 5 that enough admissible open sets exist if $h$ is small enough. Almost all open sets below will be admissible. A typical example of an admissible set is obtained as follows. Fix a subset $J$ of indices $j$ and let $G$ be the set of points where $\sum_{j \in J} \phi_{j}=1$. Then the interior of $G$ is an admissible open subset.

2.2. Discrete solution. Consider the usual bilinear form

$$
B(u, v):=\int_{\Omega} \nabla u \cdot \nabla v d x
$$

defined, for example, for $u, v \in H^{1}(\Omega)$. Assume, for the purpose of this discussion, that $\Omega$ is smooth, so that the boundary value problem (1) makes sense. Then the solution $u$ of Equation (11) satisfies

$$
B(u, v)=\left\langle g,\left.v\right|_{\partial \Omega}\right\rangle,
$$

for $v$ smooth enough. We define then the GFEM-solution of Equation (1) to be $u_{S} \in S$ such that

$$
B\left(u_{S}, v_{S}\right)=\left\langle g,\left.v_{S}\right|_{\partial \Omega}\right\rangle,
$$

and $\int_{\Omega} u_{S}(x) d x=0$. This is possible since the form $B$ is non-degenerate on the subspace $S_{0} \subset S$ consisting of functions with zero integral over $\Omega$ (recall that we are assuming, for simplicity, that $\Omega$ is connected). See also Lemma 4.1] This also shows that we need the discretization space $S$ to consist of functions that are smooth enough, which is the main reason why we are using the Generalized Finite Element Method in this paper.

\section{INTERIOR ESTIMATES FOR THE GFEM}

We continue to assume that $\Omega$ is bounded and connected. Also, we do not assume that $\Omega$ has a smooth boundary. We assume, however, that there are given $\Psi_{j}, \omega_{j}^{*} \subset \omega_{j}$, and $\phi_{j}$ be as in the previous section. In particular, they are assumed to satisfy Assumptions A, B, C, and D. This will rule out some sets $\Omega$, but any $\Omega$ with piecewise smooth boundary enjoys this property, see Section [5] Let $S=S_{G F E M}$ be the resulting Generalized Finite Element Space.

All the parameters appearing in Assumptions A-D, except $h$, will be fixed in what follows (i.e., $A, B, C_{j}, \kappa, m$, and $\sigma$ will be fixed). In particular, when we shall say that certain other constants are "independent of our choice of $h$ and GFEM-space $S$," we shall understand that all possible choices of a GFEM-space are allowed, as long as the Assumptions A-D are satisfied for an arbitrary, but fixed, choice of the parameters $A, B, C_{j}, \kappa$, and $\sigma$. In particular, our "constants" 
are allowed to depend on $A, B, C_{j}, \kappa, m$, and $\sigma$. The parameter $h$ that measures the degree of the refinement of our covering is allowed, however, to become as small as we want. Also, all constants will be independent on $N$, the number of open sets in our covering $\left\{\omega_{j}\right\}$.

All our results below remain true (with the same constants) if we replace $\Omega$ by an arbitrary admissible open subset $\Omega_{1} \subset \Omega$. (Recall that admissible open subsets were introduced in Definition 2.3.)

3.1. $H^{k}$-approximation. We shall need a basic result on the approximation of functions in $H^{k}(\Omega)$ with elements in the GFEM-space $S$, extending Theorem 2.2 Only the case $k=1$ will be needed in this section, but later on we shall need the general case.

First, let us recall the following standard lemma.

Lemma 3.1. Let $\psi_{j}$ be measurable functions defined on $\Omega$. Assume that there exists an integer $\kappa$ such that a point $x \in \Omega$ can belong to no more than $\kappa$ of the sets $\operatorname{supp}\left(\psi_{j}\right)$. Let $f=\sum_{j} \psi_{j}$. Then there exists a constant $C>0$, depending only on $\kappa$, such that $\|f\|_{H^{l}(\Omega)}^{2} \leq C \sum_{j}\left\|\psi_{j}\right\|_{H^{l}(\Omega)}^{2}$.

Proof. The inequality

$$
\left|a_{1}+a_{2}+\ldots+a_{M}\right|^{2} \leq M\left(\left|a_{1}\right|^{2}+\left|a_{2}\right|^{2}+\ldots+\left|a_{M}\right|^{2}\right)
$$

gives the desired result.

Let $k \in \mathbb{Z}_{+}$. We shall denote as usual

$$
|u|_{W^{k, \infty}(\Omega)}:=\max _{|\alpha|=k}\left\|\partial^{\alpha} u\right\|_{L^{\infty}(\Omega)}, \quad\|u\|_{W^{k, \infty}(\Omega)}:=\max _{|\alpha| \leq k}\left\|\partial^{\alpha} u\right\|_{L^{\infty}(\Omega)},
$$

$W^{k, \infty}(\Omega):=\left\{u,\|u\|_{W^{k, \infty}(\Omega)}<\infty\right\}$, and $\|\nabla \omega\|_{W^{k, \infty}(\Omega)}:=\sum_{j}\left\|\partial_{j} \omega\right\|_{W^{k, \infty}(\Omega)}$. In particular, $|u|_{W^{0, \infty}(\Omega)}=\|u\|_{W^{0, \infty}(\Omega)}=\|u\|_{L^{\infty}(\Omega)}$. We are ready now to prove the following theorem.

Theorem 3.2. Assume that, for each $j$, the local approximation spaces $\Psi_{j}$ contain (the restriction to $\omega$ ) of the degree $l-1$ polynomials. Then, for any $v \in H^{l}(\Omega)$ and any any $0 \leq k \leq l$, there exists $w \in S$ such that

$$
\|v-w\|_{H^{k}(\Omega)} \leq C h^{l-k}\|v\|_{H^{l}(\Omega)}
$$

for a constant $C$ independent of our choice of $h, S$, and $v \in S$.

Let us notice that, by taking $k=l$ in the above theorem, we immediate obtain that, using the same notation,

$$
\|w\|_{H^{l}(\Omega)} \leq C\|v\|_{H^{l}(\Omega)} .
$$

Proof. We shall use the notation and the results from [?][Chapter 4]. Let $Q_{y, f}(x)$ for any $y \in \omega_{j}^{*}$ be the Taylor polynomial of degree $l-1$ at $y$ associated to a smooth function $f$. Let then

$$
w_{j}(f)(x):=\left(\operatorname{vol}\left(\omega_{j}^{*}\right)\right)^{-1} \int_{\omega_{j}^{*}} Q_{y, f}(x) d y \in \Psi_{j}
$$

be the Taylor polynomial of degree $l-1$ averaged over $\omega_{j}^{*}$. (In the terminology of $[?]$, this is the "Taylor polynomial of order $l$ averaged over $\omega_{j}^{*}$.) The definition of 
$w_{j}(f)$ extends to any $f \in H^{l-1}(\Omega)$. Then we have the well known Bramble-Hilbert Lemma [?][Lemma 4.3.8]

$$
\left|f-w_{j}(f)\right|_{H^{k}\left(\omega_{j}\right)} \leq C h^{l-k}|f|_{H^{l}\left(\omega_{j}\right)},
$$

with a constant $C$ depending only on $\sigma$. Let $w_{j}=w_{j}(v) \in \Psi_{j}$ and $w=\sum_{j=1}^{N} \phi_{j} w_{j} \in$ $S$. Then, using also Assumption B and Lemma 3.1] we obtain

$$
\begin{aligned}
& |v-w|_{H^{s}(\Omega)}^{2} \leq C \sum_{j=1}^{N}\left|\phi_{j}\left(v-w_{j}\right)\right|_{H^{s}\left(\omega_{j}\right)}^{2} \\
& \leq C \sum_{j=1}^{N} \sum_{i=1}^{s}\left|\phi_{j}\right|_{W^{i, \infty}\left(\omega_{j}\right)}^{2}\left|\left(v-w_{j}\right)\right|_{H^{s-i}\left(\omega_{j}\right)}^{2} \leq C \sum_{j=1}^{N} \sum_{i=1}^{s} C_{i}^{2} h^{-2 i} h^{2 l-2 s+2 i}|v|_{H^{l}\left(\omega_{j}\right)}^{2} \quad \leq C \kappa h^{2 l-2 s}|v|_{H^{l}(\Omega)}^{2}
\end{aligned}
$$

Summing over $0 \leq s \leq k$ gives the desired result.

Let us also record, for further use, the following well known Poincaré-Friedrichs inequality [?], Lemma (4.3.8). (See also [?], Lemma (4.3.14), and [?], Equation (2.2), Theorem 14.1, and Theorem 15.3., or [?, ?].) The precise statement that we need is the following.

Theorem 3.3. Using the notation of Theorem 2.2, we have

$$
\|v\|_{L^{2}\left(\omega_{j}\right)} \leq C_{P} h|v|_{H^{1}\left(\omega_{j}\right)},
$$

for all $v \in H^{1}\left(\omega_{j}\right)$ satisfying $\int_{\omega_{j}} v d x=0$, where $C_{P}$ depends only on $\sigma$.

Theorems 2.2 and 3.3 lead to the estimate

$$
\left|u-u_{a p}\right|_{H^{1}(\Omega)} \leq(2 \kappa)^{1 / 2}\left(C_{1}^{2} C_{P}^{2}+C_{0}^{2}\right)^{1 / 2}\left(\sum\left\|\nabla\left(u-v_{j}\right)\right\|_{L^{2}\left(\omega_{j}\right)}^{2}\right)^{1 / 2},
$$

provided that $\int_{\omega_{j}}\left(u-v_{j}\right) d x=0$.

For $k=1$, we shall need the following consequence of Theorem 3.2 which replaces Assumption 9.5 of [?]. Define

$$
S^{<}:=S_{G F E M} \cap \mathcal{C}_{c}(\Omega) .
$$

That is, $S^{<}$consists of the elements of $S_{G F E M}$ with compact support inside $\Omega$.

Proposition 3.4. Let $\Omega_{1} \Subset \Omega$ and $\theta$ be the distance from $\partial \Omega$ to $\partial \Omega_{1}$. Then there exists $C>0$, independent of $\theta, h$, and the GFEM-space $S$, with the following property. For any $u \in H^{2}(\Omega)$ with support in $\Omega_{1}$, there exists $w \in S^{<}$such that

$$
\|u-w\|_{H^{1}(\Omega)} \leq C \theta^{-1} h\|u\|_{H^{2}(\Omega)} .
$$

Proof. Choose $w_{j}$ and $w$ as in the proof of Theorem 3.2 If $h<\theta$, then $w_{j}=0$ unless $\omega_{j}$ intersects $\Omega_{1}$, which gives that the closure of $\omega_{j}$ is completely contained in $\Omega$. Hence the support of $w$ constructed above is compact. For $h \geq \theta$, it is enough to choose $C$ large, by Theorem 3.2 
3.2. The super-approximation property. Recall the bilinear form

$$
B(u, v):=\int_{\Omega} \nabla u \cdot \nabla v d x, \quad u, v \in H^{1}(\Omega) .
$$

Our approach follows the approach from [?], as presented in [?][Section 9]. See $[?, ?, ?, ?]$ for related results on approximation in the "sup"-norm. Recall that $A \Subset B$ means that the closure of $A$ is a compact set contained in the interior of $B$. Our main goal in this section is to prove Theorem 3.12

Lemma 3.5. Let $\rho$ be a smooth function on $\omega_{j}$ and $w \in \Psi_{j}$. Then there exists $\tilde{w} \in \Psi_{j}$ such that

$$
\|\rho w-\tilde{w}\|_{H^{1}\left(\omega_{j}\right)} \leq \hat{C} h\|\rho\|_{W^{2, \infty}\left(\omega_{j}\right)}\|w\|_{H^{1}\left(\omega_{j}\right)},
$$

where $\hat{C}>0$ may depend only on the dimension $n$ (in particular, it is independent of $w, \rho$, or $j$ ).

Proof. We shall use the inner product induced from $H^{1}\left(\omega_{j}\right)$. Let $\rho \in W^{2, \infty}\left(\omega_{j}\right)$ be given.

To prove the lemma, we shall assume first that $w \in \Psi_{j}$ is a constant. Let $l$ be the degree one Taylor polynomial approximation of $\rho$ at the center of the ball $\omega_{j}^{*}$. Then $l \in \Psi_{j}$, because first order polynomials are in $\Psi_{j}$ (Assumption C) and we have

$$
\|\rho-l\|_{W^{1, \infty}\left(\omega_{j}\right)} \leq \hat{C} h\|\rho\|_{W^{2, \infty}\left(\omega_{j}\right)},
$$

with $\hat{C}>0$ a constant depending only on the dimension $n$. (This is where the condition $h \leq 1$ is used.) Choose $\tilde{w}=l w$. Then

$$
\begin{array}{r}
\|\rho w-\tilde{w}\|_{H^{1}\left(\omega_{j}\right)}=\|\rho w-l w\|_{H^{1}\left(\omega_{j}\right)} \leq\|\rho-l\|_{W^{1, \infty}\left(\omega_{j}\right)}\|w\|_{H^{1}\left(\omega_{j}\right)} \\
\leq \hat{C} h\|\rho\|_{W^{2, \infty}\left(\omega_{j}\right)}\|w\|_{H^{1}\left(\omega_{j}\right)} .
\end{array}
$$

Assume now that $w \in \Psi_{j}$ is such that $(w, 1)=0$, that is, $w$ is orthogonal in $H^{1}\left(\omega_{j}\right)$ to the subspace generated by constants. We then write

$$
\rho=\tilde{\rho}+\rho^{*},
$$

where $\tilde{\rho}$ is a constant function (say the value of $\rho$ at the center of $\omega^{*}$ ) and

$$
\left\|\rho^{*}\right\|_{L^{\infty}\left(\omega_{j}\right)} \leq \hat{C} h\|\nabla \rho\|_{L^{\infty}\left(\omega_{j}\right)}
$$

where $\hat{C}$ is a constant depending only on the dimension $n$. We shall choose then $\tilde{w}=\tilde{\rho} w \in \Psi_{j}$, which makes sense since $\Psi_{j}$ is a vector space. Then

$$
\begin{aligned}
& \|\rho w-\tilde{w}\|_{H^{1}\left(\omega_{j}\right)}=\left\|\rho^{*} w\right\|_{H^{1}\left(\omega_{j}\right)} \leq C\left\|\nabla \rho^{*}\right\|_{L^{\infty}\left(\omega_{j}\right)}\|w\|_{L^{2}\left(\omega_{j}\right)} \\
& +C\left\|\rho^{*}\right\|_{L^{\infty}\left(\omega_{j}\right)}\|w\|_{H^{1}\left(\omega_{j}\right)} \leq \hat{C} h\|\nabla \rho\|_{L^{\infty}\left(\omega_{j}\right)}\|w\|_{H^{1}\left(\omega_{j}\right)},
\end{aligned}
$$

where in the last step we have used the Poincaré-Friedrichs inequality for $\omega_{j}$ (Theorem (3.3) to estimate $\|u\|_{L^{2}\left(\omega_{j}\right)}$ and Equation (32) above to estimate the second term. Here $\hat{C}$ is again a constant that may depend only on the dimension $n$. 
For a general $w \in \Psi_{j}$, we decompose $w=w_{1}+w_{2}$ with $w_{1}$ a constant and $w_{2}$ orthogonal to the space of constants and choose $\tilde{w}_{1}$ and $\tilde{w}_{2}$ as above. Then

$$
\begin{aligned}
\left\|\rho w-\tilde{w}_{1}-\tilde{w}_{2}\right\|_{H^{1}\left(\omega_{j}\right)} \leq\left\|\rho w_{1}-\tilde{w}_{1}\right\|_{H^{1}\left(\omega_{j}\right)}+\left\|\rho w_{2}-\tilde{w}_{2}\right\|_{H^{1}\left(\omega_{j}\right)} \\
\leq \hat{C} h\|\rho\|_{W^{2, \infty}\left(\omega_{j}\right)}\left\|w_{1}\right\|_{H^{1}\left(\omega_{j}\right)}+\hat{C} h\|\rho\|_{W^{2, \infty}\left(\omega_{j}\right)}\left\|w_{2}\right\|_{H^{1}\left(\omega_{j}\right)} \\
\leq C h\|\rho\|_{W^{2, \infty}\left(\omega_{j}\right)}\|w\|_{H^{1}\left(\omega_{j}\right)} .
\end{aligned}
$$

The lemma is now proved.

An important technical step in our proof of the Theorem 3.12 is the following "super-approximation" result.

Proposition 3.6. Let $\rho \in W^{2, \infty}(\Omega)$ and $w \in S=S_{G F E M}$. Then there exists $\tilde{w} \in S$ such that

$$
\|\rho w-\tilde{w}\|_{H^{1}(\Omega)} \leq C h\|\rho\|_{W^{2, \infty}(\Omega)}\|w\|_{H^{1}(\Omega)},
$$

where $C$ is independent of $h$ and our choice of GFEM-space $S$. If $u$ has support in $\Omega_{1} \Subset \Omega$, then we can chose $\tilde{w}$ to have compact support in $\Omega$ and $C \leq C^{\prime} \theta^{-1}$, where $\theta$ is the distance from $\partial \Omega$ to $\partial \Omega_{1}$ and $C^{\prime}$ is independent of $\theta, h$, and $S$.

As explained above, the constant $C$ may depend, however, on the parameters $A$, $B, C_{j}, \kappa, m, \sigma$, and $\theta$, but is independent of $h$ or of the number $N$ of sets $\left\{\omega_{j}\right\}$.

Proof. Let

$$
w=\sum_{j=1}^{N} \phi_{j} w_{j} \in S=S_{G F E M}, \quad w_{j} \in \Psi_{j} .
$$

Let $\tilde{w}_{j}$ be the orthogonal projection of $\rho w_{j}$ onto $\Psi_{j}$ in the inner product of $H^{1}\left(\omega_{j}\right)$. Lemma 3.5 then shows that

$$
\left\|\rho w_{j}-\tilde{w}_{j}\right\|_{H^{1}\left(\omega_{j}\right)} \leq \hat{C} h\|\rho\|_{W^{2, \infty}(\Omega)}\left\|w_{j}\right\|_{H^{1}\left(\omega_{j}\right)} .
$$

Moreover, we have that $\int_{\omega_{j}}\left(\rho w_{j}-\tilde{w}_{j}\right) d x=0$ because the constant functions are in $\Psi_{j}$ and $\rho w_{j}-\tilde{w}_{j}$ is orthogonal to $\Psi_{j}$.

Let $\tilde{w}:=\sum_{j=1}^{N} \phi_{j} \tilde{w}_{j}$. Then $\left\|\nabla \phi_{j}\right\|_{L^{\infty}\left(\omega_{j}\right)} \leq C_{1} / h$ by Equation (13) and

$$
\left\|\rho w_{j}-\tilde{w}_{j}\right\|_{L^{2}\left(\omega_{j}\right)} \leq C_{P} h\left\|\rho w_{j}-\tilde{w}_{j}\right\|_{H^{1}\left(\omega_{j}\right)} \leq C_{P} \hat{C} h^{2}\|\rho\|_{W^{2, \infty}(\Omega)}\left\|w_{j}\right\|_{H^{1}\left(\omega_{j}\right)},
$$

by the Poincaré-Friedrichs inequality (Theorem 3.3), and hence

$$
\begin{gathered}
\|\rho w-\tilde{w}\|_{H^{1}(\Omega)}^{2}=\left\|\sum_{j=1}^{N} \phi_{j}\left(\rho w_{j}-\tilde{w}_{j}\right)\right\|_{H^{1}(\Omega)}^{2} \leq C \sum_{j=1}^{N}\left(\left\|\phi_{j}\right\|_{L^{\infty}\left(\omega_{j}\right)}^{2}\left\|\rho w_{j}-\tilde{w}_{j}\right\|_{H^{1}\left(\omega_{j}\right)}^{2}\right. \\
\left.\quad+\left\|\nabla \phi_{j}\right\|_{L^{\infty}\left(\omega_{j}\right)}^{2}\left\|\rho w_{j}-\tilde{w}_{j}\right\|_{L^{2}\left(\omega_{j}\right)}^{2}\right) \leq C h^{2}\|\rho\|_{W^{2, \infty}(\Omega)}^{2} \sum_{j=1}^{N}\left\|w_{j}\right\|_{H^{1}\left(\omega_{j}\right)}^{2},
\end{gathered}
$$

where for the first inequality we have used also Lemma 3.1 The result will follow now if we can prove that $\sum_{j=1}^{N}\left\|w_{j}\right\|_{H^{1}\left(\omega_{j}\right)}^{2} \leq C\|w\|_{H^{1}(\Omega)}^{2}$, for any $w=\sum_{j=1}^{N} \phi_{j} w_{j}$, as above and $C$ a constant independent of $h$ and $S$. Indeed, we have

$$
\|w\|_{H^{1}(\Omega)}^{2} \geq \sum_{j=1}^{N}\left\|w_{j}\right\|_{H^{1}\left(\omega_{j}^{*}\right)}^{2} \geq A^{2} \sum_{j=1}^{N}\left\|w_{j}\right\|_{H^{1}\left(\omega_{j}\right)}^{2},
$$


by Assumption $\mathrm{C}$ ( $A$ is the constant appearing in that assumption).

The proof of the last part is completed as in Proposition 3.4

3.3. Estimates on "discrete-harmonic" functions. We shall also need the following "inverse property," which is somewhat similar to Assumption A.3. in [?] or Assumption 9.2 in [?].

Lemma 3.7. There exists $C>0$, independent of $h, u$, and the GFEM-space $S$ such that

$$
\|w\|_{H^{j}(\Omega)} \leq C h^{i-j}\|w\|_{H^{i}(\Omega)}
$$

for all $0 \leq i \leq j \leq m$.

Recall that the constant $m$ is the fixed integer appearing in Assumptions A-D.

Proof. If $i=j$, we can take $C=1$. Let $w=\sum_{k=1}^{N} \phi_{k} w_{k}$, with $w_{k} \in \Psi_{k}$. Then Lemma 3.1 and Assumptions $\mathrm{A}-\mathrm{D}$ give

$$
\begin{aligned}
\|w\|_{H^{j}(\Omega)}^{2} & \leq \sum_{k=1}^{N} \sum_{l=0}^{j}\left\|\phi_{k}\right\|_{W^{l, \infty}\left(\omega_{k}\right)}^{2}\left\|w_{k}\right\|_{H^{j-l}\left(\omega_{k}\right)}^{2} \\
\leq \sum_{k=1}^{N} \sum_{l=0}^{j} C_{l}^{2} h^{-2 l} B^{2} h^{2 l-2 j}\left\|w_{k}\right\|_{L^{2}\left(\omega_{k}\right)}^{2} \leq C^{2} A^{2} h^{-2 j} \sum_{k=1}^{N}\left\|w_{k}\right\|_{L^{2}\left(\omega_{k}^{*}\right)}^{2} & \leq C^{2} h^{-2 j}\|w\|_{L^{2}(\Omega)}^{2} .
\end{aligned}
$$

This proves the result for $i=0$. For an arbitrary $0 \leq i \leq j$, the result follows by interpolation.

The rest of this section follows closely the approach in the paper of Nitsche and Schatz [?], relying also from the survey paper [?] (which in turn is based on the paper by Nitsche and Schatz). There are, however, some differences in the assumptions that we are using, so we include complete proofs for the convenience of the reader. For instance, the following corollary of Lemma 3.7 plays the role of Assumption A.3. in the Nitsche-Schatz article [?], respectively, of the Assumption 9.2 (Inverse assumption) in Wahlbin's article. Also, the following lemma is an analog of Lemma 5.2 of [?], respectively, of Lemma 9.1 of [?]. Recall that all the above results remain true if $\Omega$ is replaced by an admissible open subset $\Omega_{1} \subset \Omega$.

Corollary 3.8. Let $w \in S$. Then there exists a constant $C>0$ such that

$$
\|w\|_{H^{-i}(\Omega)} \leq C h^{i-j}\|w\|_{H^{-j}(\Omega)},
$$

for any $0 \leq i \leq j \leq m$.

Proof. If $i=j$ we can take $C=1$. Assume next that $i=0$. Then

$$
\|w\|_{H^{-j}(\Omega)}=\sup \frac{|(w, \phi)|}{\|\phi\|_{H^{j}(\Omega)}} \geq \frac{(w, w)}{\|w\|_{H^{j}(\Omega)}}=\frac{\|w\|_{L^{2}(\Omega)}^{2}}{\|w\|_{H^{j}(\Omega)}} \geq C^{-1} h^{j}\|w\|_{L^{2}(\Omega)},
$$

by Lemma 3.7. For the other values of $j$, the result follows by interpolation.

We shall denote $A \Subset B$ if $\bar{A}$, the closure of $A$ in $\mathbb{R}^{2}$, is a compact subset of the interior of $B$. Also, we shall denote

$$
S^{<}(A):=\left\{u \in S_{G F E M}, \operatorname{supp}(u) \Subset A\right\}
$$

for any admissible open subset $A \subset \Omega$. In particular, $S^{<}(\Omega)=S^{<}$. 
Lemma 3.9. Let $A \Subset A_{1} \Subset \Omega$ be admissible open sets and $\theta=\operatorname{dist}\left(\partial A, \partial A_{1}\right)$. Then there exists $C>0$, independent of $h, S, A$, and $A_{1}$ with the following property. If $w \in S=S_{G F E M}$ and

$$
B(w, \chi)=0, \quad \text { for all } \chi \in S^{<}\left(A_{1}\right),
$$

then, for $h$ small enough, $\|w\|_{H^{1}(A)} \leq C\|w\|_{L^{2}\left(A_{1}\right)}$, with $C$ depending only on $\theta$ and not on $A, A_{1}, h$, or $S$.

Proof. The proof is the same as the one in [?, ?], using Lemma 3.7 in place of Assumption A.3, respectively Assumption 9.2 ("Inverse assumption"), and Proposition [3.6] in place of Assumption A.2, respectively Assumption 9.1 ("Superapproximation"), of [?], respectively [?]. The constants " $C$ " below are allowed to depend on $\theta$.

Let us chose $A \Subset A_{0} \Subset A_{1}$ admissible open sets such that the distances between the boundaries of these sets are $\geq \theta / C$. This is possible if $h<c \theta$ (see Section 5 ). Also, let $\omega \in \mathcal{C}_{\mathrm{c}}^{\infty}\left(A_{0}\right)$, with $\omega=1$ on $A, \omega \geq 0$ on $A_{0}$, and $\|\omega\|_{W^{k, \infty}(\Omega)} \leq C \theta^{-k}$, for $k=0,1$. Then, by Equation (38), we obtain

$$
\begin{aligned}
\|\nabla w\|_{L^{2}(A)}^{2} \leq(\nabla w, \omega \nabla w)=(\nabla w, \nabla(\omega w)) & -(\nabla w, \nabla(\omega) w) \\
= & (\nabla w, \nabla(\omega w-\psi))+\frac{1}{2}(w,(\Delta \omega) w),
\end{aligned}
$$

where the inner products are in $L^{2}\left(A_{0}\right)$ and $\psi \in S^{<}\left(A_{0}\right)$. Proposition 3.6 then gives

which, in turn, implies

$$
\|\nabla w\|_{L^{2}(A)}^{2} \leq C h\|\omega\|_{W^{2, \infty}(\Omega)}\|w\|_{H^{1}\left(A_{0}\right)}^{2}+C\|w\|_{L^{2}\left(A_{0}\right)}^{2},
$$

$$
\|w\|_{H^{1}(A)} \leq C\left(h^{1 / 2}\|w\|_{H^{1}\left(A_{0}\right)}+\|w\|_{L^{2}\left(A_{0}\right)}\right) .
$$

We now repeat the argument for $A_{0} \Subset A_{1} \Subset \Omega$ (and $A$ replaced by $A_{0}$ and $A_{0}$ replaced by $A_{1}$ ), which gives

$$
\|w\|_{H^{1}\left(A_{0}\right)} \leq C\left(h^{1 / 2}\|w\|_{H^{1}\left(A_{1}\right)}+\|w\|_{L^{2}\left(A_{1}\right)}\right) .
$$

Combining Equations (39) and (40) and using also $h \leq 1$, we obtain

$$
\|w\|_{H^{1}(A)} \leq C\left(h\|w\|_{H^{1}\left(A_{1}\right)}+C\|w\|_{L^{2}\left(A_{1}\right)}\right) .
$$

Since $A_{1}$ is admissible, we can use Lemma 3.7 with $\Omega$ replaced with $A_{1}$ to obtain $h\|w\|_{H^{1}\left(A_{1}\right)} \leq C\|w\|_{L^{2}\left(A_{1}\right)}$, with $C$ independent of $h, w$, and the GFEM space $S$ (as long as $S$ satisfies the Assumptions A-D). Then

$$
\|w\|_{H^{1}(A)} \leq C\|w\|_{L^{2}\left(A_{1}\right)} .
$$

The proof is now complete.

We shall need the following simple estimate.

Lemma 3.10. Let $\Phi(x)=\log |x|$ if $n=2, \Phi(x)=|x|^{2-n}$, if $n \neq 2$. Let $U$ be a fixed bounded open subset of $\mathbb{R}^{n}$. Then there exists $C>0$, which depends only on $U$, such that

satisfies

$$
\Phi * u(x):=\int_{y} \Phi(x-y) u(y) d y
$$

$$
\|\Phi * u\|_{H^{l+2}(U)} \leq C\|u\|_{H^{l}(U)},
$$

for any $l \in \mathbb{R}$ and any $u \in \mathcal{C}_{c}^{\infty}(U)$. 
Proof. Let $\omega \in \mathcal{C}_{\mathrm{c}}^{\infty}\left(\mathbb{R}^{n}\right)$ be equal to 1 on $U$. Then $T u(x)=\omega(x)[\Phi *(\omega u)](x)$ is a pseudodifferential operator of order -2 with compactly supported distribution kernel. Hence it is bounded as a map $H^{l}\left(\mathbb{R}^{n}\right) \rightarrow H^{l+2}\left(\mathbb{R}^{n}\right)[?, ?]$. The statement follows by restricting $T$ to $\mathcal{C}_{\mathrm{c}}^{\infty}(U) \subset H^{l}\left(\mathbb{R}^{n}\right)$.

We define

$$
\|u\|_{H_{0}^{-s}(U)}=\sup \frac{|(u, v)|}{\|v\|_{H^{l}(U)}} \leq\|u\|_{H^{-s}(U)}, \quad 0 \neq v \in \mathcal{C}_{\mathrm{c}}^{\infty}(A)
$$

for any open set $U$, any $u \in L^{2}(U)$, and any $s>0$. We define $H_{0}^{-s}(U)$ to be the completion of $L^{2}(U)$ in the norm $\|u\|_{H_{0}^{-s}(U)}$. Then $H_{0}^{-s}(U), s>0$, identifies with the dual of $H_{0}^{s}(U)$.

We now prove the following lemma.

Lemma 3.11. We keep the notation and assumption of Lemma 3.9. In particular, we assume that $w \in S$ satisfies Equation (38). Then, for $h$ small enough,

$$
\|w\|_{L^{2}(A)} \leq C\|w\|_{H^{-m}\left(A_{1}\right)},
$$

where $C$ is a constant depending only on the distance $\theta$ from $\partial A$ to $\partial A_{1}$ and not on $A, A_{1}, h$, or $S$.

Combining Lemmata 3.9 and 3.11 we obtain

$$
\|w\|_{H^{1}(A)} \leq C\|w\|_{H^{-m}\left(A_{1}\right)}
$$

for $h$ small enough and any $w \in S$ satisfying the assumptions of Lemma 3.9

Proof. Let $A \Subset B_{0} \Subset B_{1} \Subset A_{1}$ be such that the distances between the boundaries of these sets are $\geq \theta / C$. This is possible if $h<c \theta$. Note that by Lemma 3.9 we have

$$
\|w\|_{H^{1}\left(B_{1}\right)} \leq C\|w\|_{L^{2}\left(A_{1}\right)} .
$$

For any $v \in \mathcal{C}_{\mathrm{c}}^{\infty}(A)$, let $V:=c_{n} \Phi * v \in H^{l+2}\left(B_{1}\right)$, where $c_{n}$ is chosen such that $\Delta V=v$ (see [?]). Lemma 3.10 then gives

$$
\|V\|_{H^{l+2}\left(B_{1}\right)} \leq C\|v\|_{H^{l}\left(B_{1}\right)}=C\|v\|_{H^{l}(A)}, \quad l \in \mathbb{Z}_{+},
$$

for some constant $C$ that depends only on $B_{1}$. Let $\omega \in \mathcal{C}_{\mathrm{c}}^{\infty}\left(B_{0}\right)$ with $\omega=1$ on $A$ and $\|\omega\|_{W^{k, \infty}(\Omega)} \leq C \theta^{-k}$, for $0 \leq k \leq m$. Since $\omega V \in \mathcal{C}_{\mathrm{c}}^{\infty}\left(B_{0}\right)$, we know from Proposition 3.4 that there exists $\chi \in S^{<}\left(B_{1}\right)$ such that

$$
\|\omega V-\chi\|_{H^{1}\left(B_{1}\right)} \leq C h\|\omega V\|_{H^{2}\left(B_{1}\right)} \leq C h\|V\|_{H^{2}\left(B_{1}\right)} \leq C h\|v\|_{L^{2}(A)} .
$$

Then, for any $v \in \mathcal{C}_{\mathrm{c}}^{\infty}(A)$,

$$
\begin{aligned}
(w, v)_{A}=(\omega w, v)_{A} & =\int_{A} \omega w \Delta V d x=\int_{B_{0}} \omega w \Delta V d x=\int_{B_{0}} \nabla(\omega w) \cdot \nabla V d x \\
& =\int_{B_{0}} w(2 \nabla \omega \cdot \nabla V-V \Delta \omega) d x+\int_{B_{1}} \nabla w \cdot \nabla(\omega V-\chi) d x,
\end{aligned}
$$

for any $\chi \in S^{<}\left(B_{1}\right)$, where the inner products are calculated on the indicated sets. Then, by combining Equations (46), (47), and (48), as well as Lemma 3.9] we 
obtain, for all $l \geq 0$,

$$
\begin{array}{r}
\left|(w, v)_{A}\right| \leq C\|w\|_{H_{0}^{-l-1}\left(B_{0}\right)}\|V\|_{H^{l+2}\left(B_{1}\right)}+h\|w\|_{H^{1}\left(B_{1}\right)}\|V\|_{H^{2}\left(B_{1}\right)} \\
\leq C\left(\|w\|_{H_{0}^{-l-1}\left(A_{1}\right)}+h\|w\|_{L^{2}\left(A_{1}\right)}\right)\|v\|_{H^{l}(A)},
\end{array}
$$

and hence

$$
\|w\|_{H_{0}^{-l}(A)} \leq C\left(\|w\|_{H_{0}^{-l-1}\left(A_{1}\right)}+h\|w\|_{L^{2}\left(A_{1}\right)}\right) .
$$

Let us now choose a sequence of open sets $A \Subset B_{1} \Subset B_{2} \Subset \ldots \Subset B_{m} \Subset \Omega$ with all distances between the boundaries greater or equal $c \theta$. Changing notation and iterating Equation (49), we obtain

$$
\begin{aligned}
& \|w\|_{L^{2}(A)} \leq C\left(\|w\|_{H_{0}^{-1}\left(B_{1}\right)}+h\|w\|_{L^{2}\left(B_{1}\right)}\right) \\
& \leq C\left(\|w\|_{H_{0}^{-2}\left(B_{2}\right)}+h\|w\|_{L^{2}\left(B_{2}\right)}\right) \leq \ldots \\
& \leq C\left(\|w\|_{H_{0}^{-m}\left(B_{m}\right)}+h\|w\|_{L^{2}\left(B_{m}\right)}\right) .
\end{aligned}
$$

We now repeat the above reasoning. We change notation again, so that, this time, $B_{m}$ becomes $B_{1}$, then we chose as before a sequence of open sets

$$
A \Subset B_{1} \Subset B_{2} \Subset \ldots \Subset B_{m} \Subset \Omega
$$

with all distances between the boundaries greater or equal $c \theta$. Then we iterate Equation (50), and obtain,

$$
\begin{aligned}
& \|w\|_{L^{2}(A)} \leq C\left(\|w\|_{H_{0}^{-m}\left(B_{1}\right)}+h\|w\|_{L^{2}\left(B_{1}\right)}\right) \\
& \leq C\left(\|w\|_{H_{0}^{-m}\left(B_{2}\right)}+h^{2}\|w\|_{L^{2}\left(B_{2}\right)}\right) \leq \ldots \leq C\left(\|w\|_{H_{0}^{-m}\left(B_{m}\right)}+h^{m}\|w\|_{L^{2}\left(B_{m}\right)}\right) \\
& \leq C\left(\|w\|_{H_{0}^{-m}\left(B_{m}\right)}+\|w\|_{H^{-m}\left(B_{m}\right)}\right) \leq C\|w\|_{H^{-m}\left(B_{m}\right)},
\end{aligned}
$$

where at the end we have used the inverse property $\|w\|_{L^{2}(U)} \leq h^{-m}\|w\|_{H^{-m}(U)}$ for any admissible open set $U \subset \Omega$ (see Corollary [3.8). The proof is now complete.

3.4. The interior error estimate. The following result, the main result of this section, is an analog of [?][Theorem 5.1] and of [?][Theorem 9.2].

Theorem 3.12. Let $A \Subset B \subset \Omega$ be admissible open sets. Then there exists $C>0$ with the following property. If $u \in H^{1}(\Omega)$ and $u_{S} \in S$ are such that $B\left(u-u_{S}, \chi\right)=0$ for all $\chi \in S^{<}:=S^{<}(\Omega)$, then for $h$ small enough,

$$
\left\|u-u_{S}\right\|_{H^{1}(A)} \leq C\left(\inf _{\chi \in S}\|u-\chi\|_{H^{1}(B)}+\left\|u-u_{S}\right\|_{H^{-m}(B)}\right) .
$$

The constant $C$ depends only on the distance $\theta=\operatorname{dist}(\partial A, \partial B)$ and not on $h, S$, or the sets $A$ and $B$.

Proof. Let $A \Subset A_{1} \Subset A_{2} \Subset B \subset \Omega$. Choose $\omega \in \mathcal{C}_{\mathrm{c}}^{\infty}\left(A_{2}\right)$ such that $\omega=1$ on $A_{1}$. Let $P_{1}$ be the $H^{1}(\Omega)$ orthogonal projection onto $S^{<}\left(A_{1}\right) \subset S \subset H^{1}(\Omega)$. Then on $A_{1}$

$$
u-u_{S}=\left(\omega u-P_{1}(\omega u)\right)+\left(P_{1}(\omega u)-u_{S}\right) .
$$

Then, by the general properties of orthogonal projections, we have

$$
\left\|\omega u-P_{1}(\omega u)\right\|_{H^{1}(\Omega)} \leq\|\omega u\|_{H^{1}(\Omega)} \leq C\|u\|_{H^{1}(B)} .
$$

Hence

$$
\left\|\omega u-P_{1}(\omega u)\right\|_{H^{-m}\left(A_{1}\right)} \leq\left\|\omega u-P_{1}(\omega u)\right\|_{H^{1}(\Omega)} \leq C\|u\|_{H^{1}(B)} .
$$


Let $w=P_{1}(\omega u)-u_{S}$. Then $B(w, \chi)=B\left(\omega u-u_{S}, \chi\right)=B\left(u-u_{S}, \chi\right)=0$, for all $\chi \in S^{<}\left(A_{1}\right)$, and hence $w$ satisfies the assumptions of Lemmata 3.9] and 3.11] From this, using also Equations (45), (51), and (53), we obtain

$$
\begin{array}{r}
\|w\|_{H^{1}(A)} \leq C\|w\|_{H^{-m}\left(A_{1}\right)} \leq\left\|\omega u-P_{1}(\omega u)\right\|_{H^{-m}\left(A_{1}\right)}+\left\|u-u_{S}\right\|_{H^{-m}\left(A_{1}\right)} \\
\leq\|u\|_{H^{1}(B)}+\left\|u-u_{S}\right\|_{H^{-m}\left(A_{1}\right)} .
\end{array}
$$

Equations (51] 54) then give

$$
\begin{array}{rlr}
\left\|u-u_{S}\right\|_{H^{-m}(A)} & \leq\left\|\omega u-P_{1}(\omega u)\right\|_{H^{-m}(A)}+\|w\|_{H^{-m}(A)} & \text { by } \\
& \leq C\|u\|_{H^{1}(B)}+\left\|u-u_{S}\right\|_{H^{-m}\left(A_{1}\right)} & \text { by }
\end{array}
$$

The desired result follows by replacing $u$ and $u_{S}$ with $u-\chi$ and, respectively, $u_{S}-\chi$, with $\chi$ in $S=S_{G F E M}$.

\section{Approximate solution of the Laplace EQUation With Distribution BOUNDARY CONDITIONS USING THE GFEM}

We shall consider the same setting as in the previous sections, and we shall further assume that $\Omega$ is a smooth domain. In particular, $S$ will be the GFEMspace associated to a partition of unity $\left\{\phi_{j}\right\}$ subordinated to the covering $\left\{\omega_{j}\right\}$ of $\Omega$ and local approximation spaces $\left\{\Psi_{j}\right\}$. We shall continue to assume that Assumptions $\mathrm{A}-\mathrm{D}$ are satisfied, for a fixed choice of constants $A, B, C_{j}, \kappa, m$, and $\sigma$. In particular, the "constants" below are allowed to depend on these parameters, but are not allowed to depend on $h$ or the specific choice of the GFEM-space $S$, as long as the constants above remain the same.

We shall denote by $\nu$ the outer unit normal vector to $\partial \Omega$. By $\partial_{\nu} u(x)$ we shall denote the directional derivative of a function $u$ in the direction of $\nu$, at some point $x$ on the boundary.

Let $u \in H^{1-k}(\Omega)$. We want to make precise in what sense we shall say that " $\Delta u=0$ as a distribution on $\Omega$." Recall first that the space $H^{1-k}(\Omega), k \in \mathbb{Z}_{+}$, $k>1$, was defined as the dual of $H^{k-1}(\Omega)$ (see Section 11). We shall write $\langle u, v\rangle:=$ $u(v) \in \mathbb{C}$ for any $u \in H^{1-k}(\Omega)$ and $v \in H^{k-1}(\Omega)$ for the "value of $u$ evaluated at $v$." We can hence define by duality $\partial_{j}:=-\partial_{j}^{*}: H^{1-k}(\Omega) \rightarrow H^{-k}(\Omega)$. This leads to a definition of $\Delta u \in H^{-1-k}(\Omega)$, for any $u \in H^{1-k}(\Omega)$ by

$$
\langle\Delta u, v\rangle:=\langle u, \Delta v\rangle, \quad \text { for any } v \in H^{1-k}(\Omega) .
$$

However, this turns out to be too strong a condition. Instead, we shall require

$$
\langle\Delta u, \phi\rangle:=\langle u, \Delta \phi\rangle=0, \quad \text { for any } \phi \in \mathcal{C}_{\mathrm{c}}^{\infty}(\Omega) .
$$

We shall say that $\Delta u=0$ as a distribution on $\Omega$ whenever Equation (55) is satisfied.

Typically, $u$ as above will arise as a solution of a boundary value problem, for example, as a solution of the boundary value problem (1). In [?, ?, ?] it was explained how to define the traces (or restrictions) $\left.u\right|_{\partial \Omega}$ and $\left.\partial_{\nu} u\right|_{\partial \Omega}$ for any $u \in$ $H^{1-k}(\Omega)$ satisfying $\Delta u=0$ as a distribution on $\Omega$. More generally, we define $\left.u\right|_{\partial \Omega} \in H^{1 / 2-k}(\Omega)$ and $\left.\partial_{\nu} u\right|_{\partial \Omega} \in H^{-1 / 2-k}(\Omega)$ by linearity, for $u=u_{1}+u_{2}$, where $u_{1} \in H^{1-k}(\Omega), \Delta u_{1}=0$ as a distribution on $\Omega$, and $u_{2} \in H^{1}(\Omega)$. We use this to define $B(u, v)$ by

$$
B(u, v):=-\langle u, \Delta v\rangle+\left\langle\left. u\right|_{\partial \Omega}, \partial_{\nu} v\right\rangle, \quad \text { for any } v \in H^{1+k}(\Omega),
$$


for $u=u_{1}+u_{2}$ as above. In view of Green's formula (see [?], for example), $B(u, v)=\int_{\Omega} \nabla u \cdot \nabla v d x$ if $u, v \in H^{1}(\Omega)$, as originally defined. It is not clear how to define $B(u, v)$ for arbitrary $u \in H^{1-k}(\Omega)$, since $\langle u, \Delta v\rangle$ is defined but the traces of $u$ may not defined in general.

From now on, we shall fix u such that, and

$$
\Delta u=0, \quad u \in H^{1-k}(\Omega),
$$

as a distribution on $\Omega$, where $k \in \mathbb{Z}_{+}, m+1 \geq k>0$, is also fixed. We do not assume that $\Delta u=0$ in $H^{-1-k}(\Omega)$ (i.e., we do not assume $\langle u, \Delta v\rangle=0$ for all $v \in H^{1+k}(\Omega)$, we only assume $\langle u, \Delta v\rangle=0$ for all $\left.\phi \in \mathcal{C}_{\mathrm{c}}^{\infty}(\Omega)\right)$. We shall also assume that

$$
\langle u, 1\rangle=\left\langle\left.\partial_{\nu} u\right|_{\partial \Omega}, 1\right\rangle=0 .
$$

We have the following.

Lemma 4.1. There exists a unique $u_{S} \in S$ such that $\left\langle u_{S}, 1\right\rangle=0$ and

$$
B\left(u-u_{S}, v_{S}\right)=0 \text {, }
$$

for all $v_{S} \in S$.

Proof. Let $S_{0}$ be the subspace of the GFEM-space $S$ consisting of functions $\chi_{0} \in S$ with $\int_{\Omega} \chi(x) d x=0$. The bilinear form $B$ is non-degenerate on $S_{0}$. This gives the existence of a unique $u_{S} \in S_{0}$ such that Equation (59) is satisfied for all $v_{S} \in S_{0}$. Since $S=S_{0}+\mathbb{C}$, the result follows from $B(u, 1)=B\left(u_{S}, 1\right)=0$.

We also have the following simple estimate.

Lemma 4.2. With $u$ as in Equation (57) above, we have

$$
|B(u, v)| \leq C\|u\|_{H^{1-k}(\Omega)}\|v\|_{H^{1+k}(\Omega)},
$$

for any $v \in H^{1-k}(\Omega)$ and a constant $C$ depending only on $\Omega$.

Proof. By definition, we have

$$
\begin{gathered}
|B(u, v)|=\left|-\langle u, \Delta v\rangle+\left\langle\left. u\right|_{\partial \Omega}, \partial_{\nu} v\right\rangle\right| \leq|\langle u, \Delta v\rangle|+\left|\left\langle\left. u\right|_{\partial \Omega}, \partial_{\nu} v\right\rangle\right| \\
\leq\|u\|_{H^{1-k}(\Omega)}\|\Delta v\|_{H^{-1+k}(\Omega)}+\|u\|_{H^{1 / 2-k}(\partial \Omega)}\|v\|_{H^{-1 / 2+k}(\partial \Omega)} \\
\leq C\|u\|_{H^{1-k}(\Omega)}\|v\|_{H^{1+k}(\Omega)} .
\end{gathered}
$$

This completes the proof.

We continue with more lemmata. Recall that $k \in \mathbb{Z}_{+}$.

Lemma 4.3. We have $\left\|u_{S}\right\|_{H^{1}(\Omega)} \leq C h^{-k}\|u\|_{H^{1-k}(\Omega)}$ for a constant $C$ depending only on $\Omega$.

Proof. The Poincaré-Friedrichs inequality and Lemma 4.2 give

$$
\begin{array}{r}
\left\|u_{S}\right\|_{H^{1}(\Omega)}^{2} \leq C B\left(u_{S}, u_{S}\right)=C B\left(u, u_{S}\right) \leq C\|u\|_{H^{1-k}(\Omega)}\left\|u_{S}\right\|_{H^{1+k}(\Omega)} \\
\quad \leq C h^{-k}\|u\|_{H^{1-k}(\Omega)}\left\|u_{S}\right\|_{H^{1}(\Omega)},
\end{array}
$$

where in the last inequality we have used Lemma 3.7

This gives the following corollaries.

Corollary 4.4. We have $\left\|u_{S}\right\|_{H^{1-k}(\Omega)} \leq C\|u\|_{H^{1-k}(\Omega)}$ for a constant $C$ depending only on $\Omega$. In particular, $\left\|u-u_{S}\right\|_{H^{1-k}(\Omega)} \leq C\|u\|_{H^{1-k}(\Omega)}$. 
Proof. The result is well known for $k=0$ since $u_{S}$ is the $B$-orthogonal projection of $u$ onto $S$ (see also Cèa's Lemma, [?, ?]). We shall therefore assume that $k \geq 1$.

Let $v \in H^{k-1}(\Omega)$ be arbitrary. Let $c \in \mathbb{C}$ be such that $\int_{\Omega}(v-c) d x=0$. Then we can find $V \in H^{k+1}(\Omega)$ such that

$$
-\Delta V=v-c, \int_{\Omega} V d x=0, \partial_{\nu} V=0, \quad \text { and }\|V\|_{H^{k+1}(\Omega)} \leq C\|v\|_{H^{k-1}(\Omega)},
$$

where $C$ is a constant depending only on $\Omega$. Also, chose $w \in S$ such that

$$
\|w\|_{H^{k+1}(\Omega)} \leq C\|V\|_{H^{k+1}(\Omega)} \text { and }\|V-w\|_{H^{1}(\Omega)} \leq C h^{k}\|V\|_{H^{k+1}(\Omega)} .
$$

This is possible by Theorem 3.2 Then

$$
\begin{aligned}
\left\langle u_{S}, v\right\rangle=\left\langle u_{S}, v-c\right\rangle= & -\left\langle u_{S}, \Delta V\right\rangle=-\left\langle u_{S}, \Delta V\right\rangle+\left\langle\left. u_{S}\right|_{\partial \Omega}, \partial_{\nu} V\right\rangle=B\left(u_{S}, V\right) \\
& =B\left(u_{S}, w\right)+B\left(u_{S}, V-w\right)=B(u, w)+B\left(u_{S}, V-w\right) .
\end{aligned}
$$

Using also Lemmata 4.2 and 4.3 this gives

$$
\begin{aligned}
\left|\left\langle u_{S}, v\right\rangle\right| & \leq C\|u\|_{H^{1-k}(\Omega)}\|w\|_{H^{1+k}(\Omega)}+\left\|u_{S}\right\|_{H^{1}(\Omega)}\|V-w\|_{H^{1}(\Omega)} \\
& \leq C\|u\|_{H^{1-k}(\Omega)}\|V\|_{H^{k+1}(\Omega)}+C h^{-k}\|u\|_{H^{1-k}(\Omega)} h^{k}\|V\|_{H^{k+1}(\Omega)} \\
& \leq C\|u\|_{H^{1-k}(\Omega)}\|V\|_{H^{k+1}(\Omega)} \leq C\|u\|_{H^{1-k}(\Omega)}\|v\|_{H^{k-1}(\Omega)} .
\end{aligned}
$$

This gives the result since $\left\|u_{S}\right\|_{H^{1-k}(\Omega)}:=\sup \left|\left\langle u_{S}, v\right\rangle\right| /\|v\|_{H^{k-1}(\Omega)}, v \neq 0$.

Similarly,

Corollary 4.5. We have $\left\|\left.u_{S}\right|_{\partial \Omega}\right\|_{H^{1 / 2-k}(\partial \Omega)} \leq C\|u\|_{H^{1-k}(\Omega)}$ for a constant $C$ depending only on $\Omega$. In particular, $\left\|\left.\left(u-u_{S}\right)\right|_{\partial \Omega}\right\|_{H^{1 / 2-k}(\partial \Omega)} \leq C\|u\|_{H^{1-k}(\Omega)}$.

Proof. The proof is similar to that of the previous corollary. Let $v \in H^{-1 / 2+k}(\partial \Omega)$ be arbitrary. Let $c \in \mathbb{C}$ be a constant such that $\int_{\partial \Omega} v d S=\int_{\Omega} c d x$. Then we can find a unique $W \in H^{1+k}(\Omega)$ satisfying

$$
\Delta W=c, \int_{\Omega} W d x=0, \partial_{\nu} W=v, \text { and }\|W\|_{H^{k+1}(\Omega)} \leq C\|v\|_{H^{-1 / 2+k}(\partial \Omega)},
$$

for a constant $C>0$ depending only on $\Omega$. Using also Theorem 3.2 we choose $w \in S$ such that $\|w\|_{H^{k+1}(\Omega)} \leq C\|W\|_{H^{k+1}(\Omega)}$ and $\|W-w\|_{H^{1}(\Omega)} \leq C h^{k}\|W\|_{H^{k+1}(\Omega)}$.

Then, using also $\left\langle u_{S}, 1\right\rangle=0$, we obtain

$$
\begin{aligned}
\left\langle\left. u_{S}\right|_{\partial \Omega}, v\right\rangle=\left\langle\left. u_{S}\right|_{\partial \Omega}, \partial_{\nu} W\right\rangle=\left\langle u_{S}, \Delta W\right\rangle+B\left(u_{S}, W\right)=B\left(u_{S}, W\right) & \\
& =B\left(u_{S}, w\right)+B\left(u_{S}, W-w\right)=B(u, w)+B\left(u_{S}, W-w\right) .
\end{aligned}
$$

Using Lemmata 4.2 and 4.3, we then obtain

$$
\begin{gathered}
\left|\left\langle\left. u_{S}\right|_{\partial \Omega}, v\right\rangle\right| \leq C\|u\|_{H^{1-k}(\Omega)}\|w\|_{H^{1+k}(\Omega)}+\left\|u_{S}\right\|_{H^{1}(\Omega)}\|W-w\|_{H^{1}(\Omega)} \\
\leq C\|u\|_{H^{1-k}(\Omega)}\|W\|_{H^{1+k}(\Omega)}+C h^{-k}\|u\|_{H^{1-k}(\Omega)} h^{k}\|W\|_{H^{1+k}(\Omega)} \\
\leq C\|u\|_{H^{1-k}(\Omega)}\|W\|_{H^{1+k}(\Omega)} \leq C\|u\|_{H^{1-k}(\Omega)}\|v\|_{H^{-1 / 2+k}(\partial \Omega)}
\end{gathered}
$$

which completes the proof in view of the definition of $\left\|u_{S}\right\|_{H^{1 / 2-k}(\Omega)}$.

We complete our sequence of estimates with the following result. 
Proposition 4.6. Let $k+\gamma \leq m+1, k, \gamma \in \mathbb{Z}_{+}$. Assume that each local approximation space $\Psi_{j}$ contains the polynomials of degree $1+k+\gamma$. Then the error $u-u_{S}$ satisfies

$$
\left\|u-u_{S}\right\|_{H^{1-k-\gamma}(\Omega)} \leq C h^{\gamma}\|u\|_{H^{1-k}(\Omega)},
$$

with a constant $C$ independent of $h$ or the GFEM-space $S$, but possibly depending on $\Omega$ and the parameters $A, B, C_{j}, \kappa, \sigma$, and $m$.

Proof. Let $v \in H^{-1+k+\gamma}(\Omega)$ be arbitrary. Let $c$ be a constant such that $\langle v-c, 1\rangle=$ 0 . Then there exists a unique $V \in H^{1+k+\gamma}(\Omega)$ such that

$$
-\Delta V=v-c, \int_{\Omega} V d x=0, \partial_{\nu} V=0, \text { and }\|V\|_{H^{1+k+\gamma}(\Omega)} \leq C\|v\|_{H^{-1+k+\gamma}(\Omega)},
$$

for a constant $C>0$ depending only on $\Omega$.

Then, for any $w \in S$,

$$
\begin{array}{r}
\quad\left\langle u-u_{S}, v\right\rangle=\left\langle u-u_{S}, v-c\right\rangle=-\left\langle u-u_{S}, \Delta V\right\rangle \\
=-\left\langle u-u_{S}, \Delta V\right\rangle+\left\langle\left.\left(u-u_{S}\right)\right|_{\partial \Omega}, \partial_{\nu} V\right\rangle=B\left(u-u_{S}, V\right)=B\left(u-u_{S}, V-w\right) \\
=-\left\langle u-u_{S}, \Delta(V-w)\right\rangle+\left\langle\left.\left(u-u_{S}\right)\right|_{\partial \Omega}, \partial_{\nu} w\right\rangle .
\end{array}
$$

Using Theorem 3.2 we chose $w \in S$ such that $\|w\|_{H^{1+k+\gamma}(\Omega)} \leq C\|V\|_{H^{1+k+\gamma}(\Omega)}$ and $\|V-w\|_{H^{1+k}(\Omega)} \leq C h^{\gamma}\|V\|_{H^{1+k+\gamma}(\Omega)}$. In particular,

$$
\left\|\partial_{\nu} w\right\|_{H^{-1 / 2+k}(\partial \Omega)}=\left\|\partial_{\nu}(V-w)\right\|_{H^{-1 / 2+k}(\partial \Omega)} \leq h^{\gamma}\|V\|_{H^{1+k+\gamma}(\Omega)} .
$$

From $\|V\|_{H^{1+k+\gamma}(\Omega)} \leq C\|v\|_{H^{-1+k+\gamma}(\Omega)}$, Corollaries 4.4 and 4.5] and Equation (61), we then obtain,

$$
\begin{aligned}
&\left|\left\langle u-u_{S}, v\right\rangle\right| \leq\left\|u-u_{S}\right\|_{H^{1-k}(\Omega)}\|V-w\|_{H^{1+k}(\Omega)}+\left\|u-u_{S}\right\|_{H^{1-k}(\Omega)}\left\|\partial_{\nu} w\right\|_{H^{-1 / 2+k}(\partial \Omega)} \\
& \leq C h^{\gamma}\|u\|_{H^{1-k}(\Omega)}\|v\|_{H^{-1+k+\gamma}(\Omega)}
\end{aligned}
$$

The proof is complete.

Theorem 3.12 and Proposition 4.6 then give the following result, which is the main result of this paper.

Recall, for the following theorem, that $S=S_{G F E M}$ is the GFEM-space associated to a partition of unity satisfying Assumptions A-D. Also, recall that we have fixed $u \in H^{1-k}(\Omega)$ satisfying $\langle u, \Delta \phi\rangle=0$ for all $\phi \in \mathcal{C}_{\mathrm{c}}^{\infty}(\Omega)$ and that $u_{S}$ is the GFEM-approximation of $u$ (i.e., given by Lemma 4.1).

Proposition 4.7. Assume the local approximation spaces $\Psi_{j}$ contain the polynomials of degree $k+\gamma+1$ and let $A \Subset B \Subset \Omega$ be admissible open subsets. Then for any $-1+k \leq-1+k+\gamma \leq m$ and any $l \geq 1, k, \gamma \in \mathbb{Z}_{+}$, we have

$$
\left\|u-u_{S}\right\|_{H^{1}(A)} \leq C h^{l}\|u\|_{H^{l+1}(B)}+C h^{\gamma}\left\|u-u_{S}\right\|_{H^{-1+k+\gamma}(B)} .
$$

Proof. This follows from Theorem 3.12 and from

$$
\inf _{\chi \in S}\|u-\chi\|_{H^{1}(B)} \leq C h^{l}\|u\|_{H^{l+1}(B)} .
$$

By taking $l=\gamma$ and using also Proposition 4.6 and Equation (31), we obtain 
Theorem 4.8. Assume the local approximation spaces $\Psi_{j}$ contain the polynomials of degree $k+\gamma+1$ and let $A_{0} \Subset \Omega$ be an admissible open subset. Then for any $-1+k \leq-1+k+\gamma \leq m, k, \gamma \in \mathbb{Z}_{+}$, we have

$$
\left\|u-u_{S}\right\|_{H^{1}\left(A_{0}\right)} \leq C h^{\gamma}\|u\|_{H^{1-k}(\Omega)} .
$$

The constant $C$ above is independent of $h$ and $S$, but may depend on the parameters $A, B, C_{j}, \kappa, \sigma, l, \gamma$, and $m$, as well as on $\theta$, the distance between $\partial A_{0}$ and $\partial \Omega$.

\section{Polynomial local approximation spaces}

In this section we shall verify that the Assumptions A-D are verified if we choose $\Psi_{j}$ to be the space of (restrictions to $\omega_{j}$ of) polynomials of degree $\leq m, m \geq 1$. Most results of this section are either elementary or well known. We include them nevertheless for the benefit of the reader and for completeness.

In this section, the set of polynomials of degree $m$ will be denoted $\mathcal{Q}_{m}$. Also, for any ball $B$ of radius $r$, we shall denote by $t B$ the ball with the same center as $B$ and radius $t r$.

Lemma 5.1. There exists a constant $C>0$, depending only on $n, m$, and $t>0$, such that for any ball $B \subset \mathbb{R}^{n}$ and any $Q \in \mathcal{Q}_{m}$, we have $\|Q\|_{L^{2}(t B)} \leq C\|Q\|_{L^{2}(B)}$.

Proof. For any fixed $B, Q \mapsto\|Q\|_{L^{2}(t B)}$ and $P \mapsto\|Q\|_{L^{2}(B)}$ are two norms on the finite dimensional space $\mathcal{Q}_{m}$ of polynomials of degree $\leq m$, and hence they are equivalent. This gives the result, except the independence of $C$ on $B$. But all balls are affine equivalent and the $L^{2}$-norm is scaled by the (square root of the) determinant of the matrix of the affine transformation. Thus the constant $C$ can be chosen to be the same for all balls $B$.

This gives immediately the following corollary.

Corollary 5.2. There exists a constant $C>0$, depending only on $t>0, n$, and $m$, such that for any ball $B \subset \mathbb{R}^{n}$ and any polynomial $Q \in \mathcal{Q}_{m}$, we have $|Q|_{H^{l}(t B)} \leq C|Q|_{H^{l}(B)}$ and $\|Q\|_{H^{l}(t B)} \leq C\|Q\|_{H^{l}(B)}, 0 \leq l \leq m$.

Proof. Use Lemma 5.1 for all derivatives $Q^{(\alpha)}$, where $\alpha$ is a multi-index such that $|\alpha|=l$ or $|\alpha| \leq l$.

We now establish to the following "inverse property."

Lemma 5.3. There exists a constant $C>0$, depending only on $m, \alpha$, and $n$ such that $\left\|Q^{(\alpha)}\right\|_{L^{2}(B)} \leq C r^{l-|\alpha|}\|Q\|_{H^{l}(B)}$ for any $l \leq|\alpha| \leq m$, any $Q \in \mathcal{Q}_{m}$, and any ball $B$ of radius $r$.

Proof. Let us prove first the result for $l=0$. That is, we need to prove that $\left\|Q^{(\alpha)}\right\|_{L^{2}(B)} \leq C r^{-|\alpha|}\|Q\|_{L^{2}(B)}$.

Let $B_{1}=B_{1}(0)$ be the unit ball centered at 0 . Then $Q \mapsto\left\|Q^{(\alpha)}\right\|_{L^{2}\left(B_{1}\right)}$ is a semi-norm on $\mathcal{Q}_{m}$, the space of polynomials of degree at most $m$, and hence it is bounded by the norm $Q \mapsto\|Q\|_{L^{2}\left(B_{1}\right)}$. Thus $\left\|Q^{(\alpha)}\right\|_{L^{2}\left(B_{1}\right)} \leq C_{1}\|Q\|_{L^{2}\left(B_{1}\right)}$. Let $L$ be an affine transformation mapping $B_{1}$ onto the ball $B$ of radius $r$ consisting of the composition of a translation and a dilation of ratio $r$. Then

$$
\begin{array}{r}
\left\|Q^{(\alpha)}\right\|_{L^{2}(B)}=\operatorname{det}(L)^{1 / 2}\left\|Q^{(\alpha)} \circ L\right\|_{L^{2}\left(B_{1}\right)}=\operatorname{det}(L)^{1 / 2} r^{-|\alpha|}\left\|(Q \circ L)^{(\alpha)}\right\|_{L^{2}\left(B_{1}\right)} \\
\leq C_{1} \operatorname{det}(L)^{1 / 2} r^{-|\alpha|}\|Q \circ L\|_{L^{2}\left(B_{1}\right)}=C r^{-|\alpha|}\|Q\|_{L^{2}(B)},
\end{array}
$$


for any $Q \in \mathcal{Q}_{m}$.

Assume now that $|\alpha| \geq l>0$. Choose $\beta \leq \alpha,|\beta|=l$. Then

$$
\left\|D^{\alpha-\beta} D^{\beta} Q\right\|_{L^{2}(B)} \leq C r^{-|\alpha-\beta|}\left\|D^{\beta} Q\right\|_{L^{2}(B)} \leq C r^{l-|\alpha|}\|Q\|_{H^{l}(B)} .
$$

This completes the proof.

The relevant "inverse property" now follows.

Proposition 5.4. There exists a constant $C>0$, depending only on $\sigma, m, \alpha$, and $n$ such that $\left\|Q^{(\alpha)}\right\|_{L^{2}(\Omega)} \leq C r^{l-|\alpha|}\|Q\|_{H^{l}(B)}$ for any $l \leq|\alpha| \leq m$, any $Q \in \mathcal{Q}_{m}$, any ball $B$ of radius $r$, and any convex set $\Omega$ contained in $\sigma^{-1} B$.

Proof. This follows from Corollary 5.2 and Lemma 5.3

We now prove the following elementary lemma.

Lemma 5.5. For any $A \Subset \Omega$ and any $k \in \mathbb{Z}_{+}$, we can construct admissible open sets $A=: B_{0} \Subset B_{1} \Subset B_{2} \Subset \ldots \Subset B_{k} \Subset B_{k+1}:=\Omega$ such that $\hat{C} d i s t\left(\partial B_{j}, \partial B_{j}\right) \geq$ $\theta / k$, where $\theta:=\operatorname{dist}(\partial A, \partial \Omega)$, provided that $\hat{C} h<\theta$, where $\hat{C}$ depending on $n$ only (in particular, $\hat{C}$ is independent of $h$ ).

Proof. Take $k=1$, for simplicity. The general result is proved similarly or by iterating this case. Let $\hat{C}=4$. Let $U$ be the union of all open sets $\omega_{j}$ at distance at most $\theta / 4$ from $A$. Let $J$ be the set of indices $j$ such that $\phi_{j} \neq 0$ on $U$ and let $G$ be the set where $\sum_{j \in J} \phi_{j}=1$. We then define $B_{1}$ to be the interior of $G$.

5.1. Partition of Unity. We show in this section that, for a suitable set $\Omega$, we can choose a family of partitions of unity $\left\{\phi_{j}\right\}$, subordinated to the covering $\left\{\omega_{j}\right\}$, with $h \rightarrow 0$ but with all the other constants fixed. The proof of the following theorem is not constructive. A constructive proof, suitable for numerical implementation, will be included in a forthcoming paper where we will also discuss the numerical implementation of the GFEM for boundary value problems with distributional data.

Theorem 5.6. Let $\Omega$ be a bounded open set with piecewise $C^{1}$-boundary. Then there exist constants $A, B, C_{j}, \kappa, m$, and $\sigma$, such that, for any small enough $h>0$, we can construct a partition of unity $\left\{\phi_{j}\right\}$ subordinated to the covering $\left\{\omega_{j}\right\}$ and satisfying the Assumptions A-D of Section (2) for the given value of the parameters $A, B, C_{j}, \kappa, m, \sigma$, and $h$.

Proof. Let us first construct the covering $\left\{\omega_{j}\right\}$ and the subsets $\left\{\omega_{j}^{*}\right\}$. The index $j$ will belong to a set of points of $\Omega$, the centers of those balls. (So $\omega_{j}$ and $\omega_{j}^{*}$ will have the same center, namely $j$.)

Let $\epsilon>0$ be small enough such that, for any $y \in \Omega$ satisfying $\operatorname{dist}(y, \partial \Omega)<\epsilon$, there exists a unique $z \in \partial \Omega$ with $\operatorname{dist}(y, \partial \Omega)=\operatorname{dist}(y, z)$. Let $\Gamma_{r} \subset \Omega$ be the set of points at distance $r, r<\epsilon / 2$ to $\partial \Omega$. Then $\Gamma_{r}$ will be a piecewise $C^{1}$ curve, bounding a domain diffeomorphic to $\Omega$. Choose on $\Gamma_{r}$ a maximal set of points $X_{h}$ containing the vertices of $\Gamma_{r}$ and at distance at least $r / 2$ from one another. The maximality of $X_{h}$ then guarantees that the distance between any two consequtive points in $X_{h}$ is at most $r$. Let

$$
f(r):=\sup _{y \in \partial \Omega} \operatorname{dist}\left(y, \Gamma_{r}\right)
$$

A geometric argument based on the assumption that $\partial \Omega$ is $C^{1}$ then shows that $\lim f(r) / r=\mu$, as $r \rightarrow 0$, with $\mu$ finite. Let then $\sigma^{-1}>\max \{4(\mu+1), 8\}$ and 
$h:=r \sigma^{-1} / 2$. This guarantees that the balls with centers in $X_{h}$ and diameter $h$ will cover the region between $\Gamma$ and $\partial \Omega$. Let $Y_{h}$ be a maximal subset of $\Omega$ containing $X_{h}$ and such that any point in $Y_{h}$ is at distance at least $r$ to the boundary $\partial \Omega$ and at distance at least $r / 2$ from any other point in $Y_{h}$. The choice of $Y_{h}$ shows that the balls $\omega_{j}^{*}$ of diameter $\sigma h=r / 2$ and center at the points $Y_{h}$ will be disjoint, whereas the balls $\omega_{j}$ with the same centers and diameter $h$ will cover the interior of $\Gamma$.

Let us now construct the partition of unity $\phi_{j}$. Let $1 \geq \psi(t) \geq 0, t \geq 0$ be a smooth function such that $\psi(t)=1$ if $t \leq \sigma$ and $\psi(t)=0$ if $t \geq 1$. Also, let $\zeta(t) \geq 0$ be a smooth function such that $\zeta(t)=0$ if $t \leq 1$ and $1 \geq \zeta(t)>0$ if $t>1$ and $\zeta(t)=1$ if $t \geq 2$. Define then

$$
\eta_{j}(x)=\psi(\operatorname{dist}(x, j) / h) \prod_{j^{\prime} \neq j} \zeta\left(\operatorname{dist}\left(x, j^{\prime}\right) / h\right) .
$$

Let $\eta(x)=\sum_{j} \eta_{j}(x)$ and $\phi_{j}(x)=\eta_{j}(x) / \eta(x)$. We observe that the number of factors $\neq 1$ in the definition of $\eta_{j}$ is bounded by a constant independent of $x, j$, and any of the choices above. Therefore $\left\{\phi_{j}\right\}$ is our desired partition of unity.

Some assumptions on the domain $\Omega$ in the above theorem are necessary, as shown by the following remark.

Remark 5.7. The non-Lipschitz domain

$$
\Omega_{c}:=\left\{(x, y),-x^{2} \leq y \leq x^{2}, x^{2}+y^{2} \leq 1, x \geq 0\right\}
$$

will have no covering $\left\{\omega_{j}\right\}$ satisfying the Assumptions A-D.

The University of Texas at Austin, Institute for Computational Engineering and Sciences, Austin, TX 78712-0027

E-mail address: Babuska@ticam.utexas.edu

Pennsylvania State University, Math. Dept., University Park, PA 16802

E-mail address: nistor@math.psu.edu 